Project Report

\title{
Feeling Safe during Intensive Care: Protocol of a Pilot Study on Therapeutic Suggestions of Safety under Hypnosis in Patients with Non- Invasive Ventilation
}

\author{
Barbara Schmidt ${ }^{1,}{ }^{*}$, Teresa Deffner ${ }^{2}$, Jenny Rosendahl ${ }^{3}$
}

1. Friedrich Schiller University Jena, Institute of Psychology, Am Steiger 3, Haus 1, 07743 Jena, Germany; E-Mail: schmidt.barbara@uni-jena.de

2. Jena University Hospital, Department of Anesthesiology and Intensive Care Medicine, Am Klinikum 1, 07747 Jena, Germany; E-Mail: teresa.deffner@med.uni-jena.de

3. Jena University Hospital, Institute of Psychosocial Medicine and Psychotherapy, Stoystraße 3, 07740 Jena, Germany; E-Mail: jenny.rosendahl@med.uni-jena.de

* Correspondence: Barbara Schmidt; E-Mail: schmidt.barbara@uni-jena.de

Academic Editor: Giuseppe De Benedittis

Special Issue: Hypnosis: from Neural Mechanisms to Clinical Practice

OBM Integrative and Complementary Medicine

2020, volume 5 , issue 2

doi:10.21926/obm.icm.2002025
Received: December 11, 2019

Accepted: April 22, 2020

Published: April 28, 2020

\begin{abstract}
Patients in the intensive care unit are confronted with an extreme situation that may cause stress and anxiety. The negative experiences may cause patients to suffer for the long-term after leaving the intensive care unit. One such stressor in the intensive care unit is noninvasive ventilation. Positive therapeutic suggestions under hypnosis can help patients in intensive care to enhance their well-being by helping them to deal with specific stress like being ventilated. The aim of our study is to assess the effects of therapeutic suggestions of safety under hypnosis in patients on non-invasive ventilation during intensive care. We present a pre-post study design to evaluate the effects of standardized safety suggestions under hypnosis carried out in a face-to-face setting. We include non-invasively ventilated patients treated in the intensive care unit who must be able communicate and be oriented
\end{abstract}

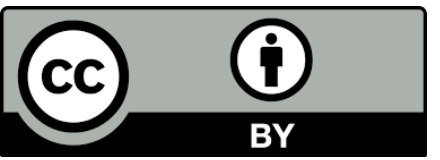

(C) 2020 by the author. This is an open access article distributed under the conditions of the Creative Commons by Attribution License, which permits unrestricted use, distribution, and reproduction in any medium or format, provided the original work is correctly cited. 
toward all dimensions, i.e., person, place, time, and particularly, situation. Patients rate their subjective feelings of valence, arousal, anxiety, as well as the aversiveness of the breathing mask, before and after receiving therapeutic suggestions of safety under hypnosis. We further record physiological parameters like heart rate, blood pressure, breathing rate, and blood oxygen level before, during, and after providing the suggestions under hypnosis. We test the effectiveness of therapeutic suggestions of safety under hypnosis in non-invasively ventilated patients during treatment in the intensive care unit. Psychological interventions such as positive suggestions under hypnosis can help to reduce acute mental distress. Patients in intensive care are in an extreme situation accompanied by stress and anxiety and often suffer from negative long-term complications; therefore, our study addresses an existential need in critical care. We thus provide evidence that positive suggestions under hypnosis increase psychological well-being and reduce the impact of stressors, such as being ventilated in the intensive care unit.

\section{Keywords}

Intensive care unit; emotional well-being; non-invasive ventilation; safe place imagination; physiological parameters; therapeutic suggestions; hypnosis

\section{Introduction}

A patient in the intensive care unit often undergoes negative experiences like pain, fear of death, being dependent on strangers, and being in an unfamiliar environment that is incomprehensible [1]. Patients in the intensive care unit are thus in an extreme situation that causes symptoms of stress, anxiety, and depression [2]. Negative experiences in the intensive care unit can often lead to long-term negative consequences [3]. One of the specific stressors in the intensive care unit, often caused by medical treatment, is non-invasive mechanical ventilation, wherein the breathing mask is positioned over the patient's nose and mouth with considerable pressure. Non-invasive ventilation primarily supports breathing, but many patients perceive the breathing mask as very uncomfortable, threatening, and stressful [4]. Altogether, patients in the intensive care unit are confronted with a generally threatening situation and are additionally exposed to specific stressors like non-invasive ventilation. Figure 1 illustrates the patient's situation in the intensive care unit with non-invasive ventilation. 


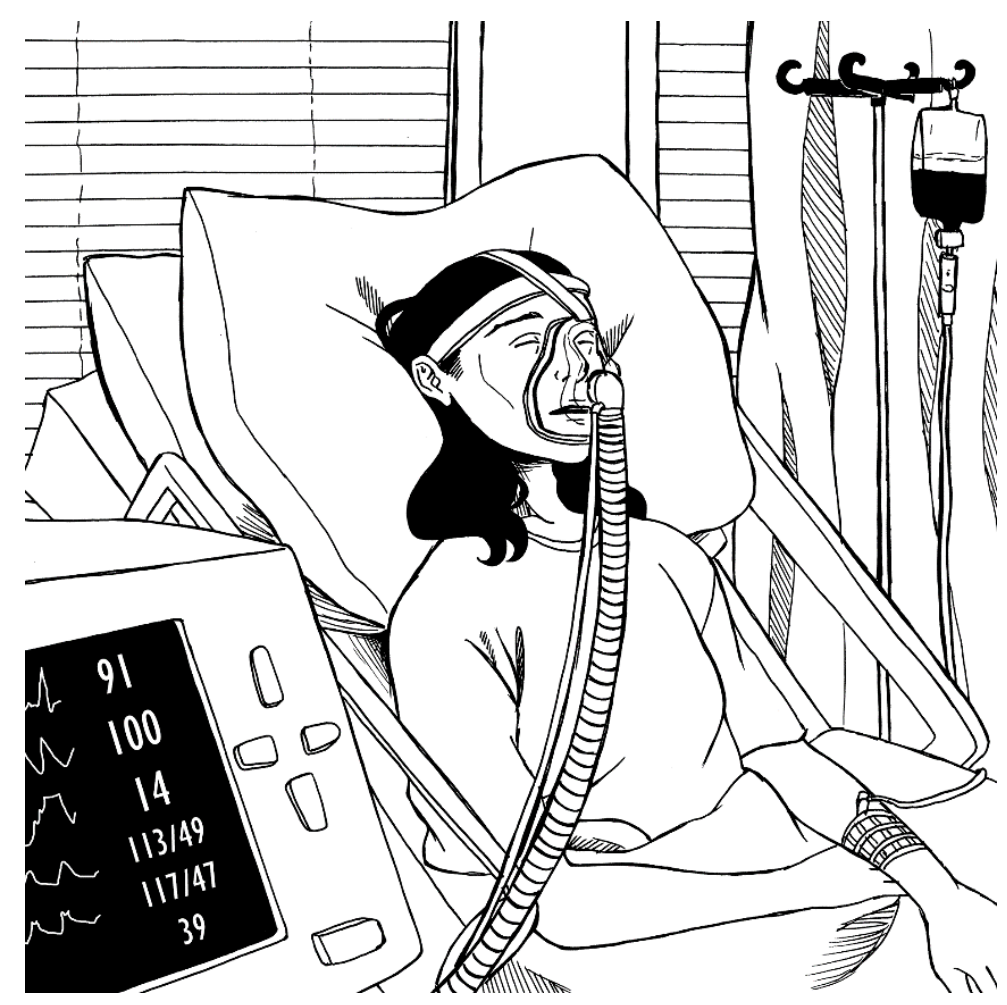

Figure 1 Illustration of a patient with non-invasive ventilation in the intensive care unit. Drawn by Sophie Elschner, https://sophssketchpad.wordpress.com/

The suggestion of a safe place is commonly made to reduce anxiety and stress [5]. Participants are told to imagine that they are in a safe place where they feel protected and calm. Evidence from hypnosis studies shows that being in a hypnotic state enhances the effects of suggestions [6]. Suggestions also work better when patients are highly suggestible. While suggestibility is a stable trait with considerable retest reliability [7], there are specific circumstances that increase suggestibility. For example, suggestibility increases in highly threatening and stressful situations [8]. As we indicated before, being in the intensive care unit is a highly threatening and stressful situation; therefore, we predict that these suggestions work very well in the intensive care unit. To achieve better results, we decided to present the suggestions after inducing a hypnotic state [6] and tested whether an intervention increases participants' general well-being and reduces the aversiveness of non-invasive ventilation. Developing such an intervention helps to improve patients' well-being immediately and prevents negative long-term consequences.

Therapeutic suggestions have positive effects on patients in the intensive care unit [9]. Previous studies demonstrated that after receiving positive therapeutic suggestions, patients could leave the intensive care unit sooner and got independent of mechanical ventilation faster [10, 11]. Moreover, patients took lower doses of benzodiazepines and opioids when they received positive therapeutic suggestions [12]. However, the existing studies focused primarily on objective medical recordings. In our study, we focus on subjective psychological outcomes like a decrease in anxiety and aversiveness ratings of the breathing mask.

We developed a suggestion text that addresses two issues. First, we include the suggestion of a safe place to induce a feeling of general well-being. Maslow [13] significantly pointed out that the need for safety is existential; therefore, we aimed at increasing the feeling of safety via our suggestion of a safe place during hypnosis. Second, we address the sensations accompanied by 
non-invasive ventilation and suggest the comfort and helpfulness of the ventilation mask. We use hypnotic suggestions to increase immediate well-being and post-hypnotic suggestions to reduce the aversiveness of the breathing mask in future treatment.

In a pilot trial using a pre-post design, we measure the effectiveness of therapeutic suggestions of safety during hypnosis in non-invasively ventilated patients. To measure the effectiveness, we use subjective ratings of valence, arousal, anxiety, and aversiveness of the breathing mask. We also record physiological responses during the intervention.

\section{Materials and Methods}

\subsection{Study Design and Setting}

This study uses a pre-post design to evaluate the effects of intervention, including therapeutic suggestions of safety during hypnosis. Patients are recruited at the Jena University Hospital, Germany, and are contacted bed-side while being treated in the intensive care unit. The intervention is delivered by students supervised by the first author of this manuscript, who is trained in hypnosis and is a member of the Milton Erickson Society for Clinical Hypnosis.

\subsection{Eligibility Criteria}

We include adult (18+ years) patients who are treated in the intensive care unit and undergo non-invasive ventilation. As we want patients to indicate their subjective ratings, we only include patients who are able to communicate and who are fully oriented, as assessed via the Glasgow Coma Scale [14]. We select patients with a Glasgow Coma Scale score of at least 14. We exclude patients who get non-invasive ventilation for the first time as they cannot rate the aversiveness of the breathing mask before the intervention and patients who do not understand German or who are deaf.

\subsection{Procedure}

Before starting with our intervention, we ask patients about their current mood using the valence and arousal scales of the Self-Assessment Manikin [15]. We also measure their current state of anxiety using the Faces Anxiety Scale [16]. To assess the aversiveness of the breathing mask, we use a visual analog scale ranging from 9 (unpleasant and disturbing) to 1 (pleasant and supporting). We select patients who have problems with non-invasive ventilation, as indicated by an aversiveness pre-score of at least 3. To measure the physiological responses to our intervention, we use physiological parameters recorded in the intensive care unit by default, including heart rate, blood pressure, breathing rate, and blood oxygen level. To assess the timing of our intervention, we record a video with a monitor showing the patient's physiological responses and the voice of the hypnotist providing the intervention.

After the breathing mask is applied, we start with the intervention. The whole intervention takes nearly $15 \mathrm{~min}$. Accordingly, our intervention targets non-invasive ventilation periods of about $15 \mathrm{~min}$. First, we induce hypnosis. As there are several possible disturbing factors in the intensive care unit like people walking around and talking, unknown odors, and beeping noises from monitors, we include them in the hypnosis induction. For example, we tell patients that the 
beeping sounds indicate the presence of people around who take good care so that the patients can relax completely. We tell patients to focus on their breathing and how the breathing mask helps them to breathe. At the end of the hypnosis induction, we count from one to ten and tell patients that with every number, they get deeper into the hypnotic state. Then, we suggest that the participants are at a safe place. We invite the patients to imagine a place where they had been earlier and felt well and safe. Next, we count from one to ten and tell the patients that they feel safer with every number. We then provide the post-hypnotic suggestion that the breathing mask feels helpful and that in the future, whenever they feel the pressure of the breathing mask again, they will experience the same feeling of safety like they are currently experiencing. This helps patients to transfer the feeling of safety in their daily treatment. Finally, we lead patients out of the hypnotic state by counting from ten to one and tell patients that with every number, they get back slowly into a normal waking state. To assess the effect of the intervention, we again ask the patients about their current state of valence and arousal, using the scale of Self-Assessment Manikin [15] and their current state of anxiety, using the Faces Anxiety Scale [16]. The patients rate the aversiveness of the breathing mask again on a visual analog scale ranging from 1 to 9.

\subsection{Outcomes}

Primary outcomes are patients' valence and arousal ratings, measured via the Self-Assessment Manikin [15] and patients' anxiety ratings, measured via the Faces Anxiety Scale [16]. Secondary outcomes are breathing mask aversiveness assessed via a visual analog scale and the physiological parameters, including heart rate, blood pressure, breathing rate, and blood oxygen level.

\subsection{Sample Size}

We estimated the effect size for the effect of hypnotic suggestions on subjective experiences in clinical settings based on a meta-analysis by Tefikow et al. [17] as Cohen's $d=0.5$. We computed the necessary sample size to detect an effect of this size in a within-subjects design with alpha = 0.05 and statistical power of 0.9 using the program $\mathrm{G}^{*}$ power [18]. The computed sample size was 36 , so we plan to test 40 participants to account for possible drop-outs.

\subsection{Data Analysis}

Primary and secondary outcomes will be analyzed by statistically comparing pre- and postmeasures. To analyze the physiological responses to our intervention, we will use the video that we recorded during the intervention and set event markers to assess how specific parts of the text affected patients' physiological parameters. Pre-post effects will be computed as standardized mean differences (Cohen's $d$ ) with 95\% confidence intervals.

\subsection{Ethical Considerations}

All eligible participants are informed verbally about the study aims, content, procedure, and duration. Participation is voluntary at any time, and the participants were informed about the same and the choice to interrupt or prematurely terminate study participation without giving reasons. We obtained ethical approval for our study from the ethics committee of the FriedrichSchiller University, Jena, Germany (\#2019-1463). 


\section{Expected Results}

We expect more positive valence ratings and a decline in arousal ratings after the intervention. Further, we predict that anxiety ratings and aversiveness ratings of the breathing mask significantly decrease after the intervention. Considering the physiological parameters, we predict that heart rate, blood pressure, and breathing rate decrease due to the intervention. Overall, we expect that after the intervention, patients show better compliance, perceive the breathing mask as less disturbing, and show less anxiety on being ventilated.

\section{Discussion}

This study aims to provide the first evidence of a treatment approach particularly designed for patients experiencing stress during non-invasive mechanical ventilation in intensive care. Noninvasive ventilation is one of many stressors in intensive care because of the pressure of the breathing mask and the perceived lack of control, causing feelings of helplessness, anxiety, and discomfort $[1,2,4]$. We provide here an intervention using hypnosis that may help to increase patients' general well-being and also specifically help in improving the perception of non-invasive mechanical ventilation.

Our study is a pilot trial to assess the applicability and to provide the first evidence on treatment effects, and randomized-controlled studies will be needed in the future to test the efficacy of the intervention against a suitable control group in a sufficiently sized sample of patients. Our study is the first attempt to focus on patients' subjective well-being besides the objective medical parameters in a largely technological setting of intensive care. In order to prevent acute anxiety and stress as well as negative long-term consequences, it is necessary to focus on the psychological well-being of the patient in the intensive care unit. Therefore, our study addresses an existential need in critical care.

\section{Acknowledgments}

We thank Lisa Planer, Ramona Schunke and Jana Schneider for their help with the study.

\section{Author Contributions}

All authors wrote the paper.

\section{Competing Interests}

The authors have declared that no competing interests exist.

\section{References}

1. Stein-Parbury J, McKinley S. Patients' experiences of being in an intensive care unit: A selected literature review. Am J Crit Care. 2000; 9: 20-27.

2. Peris A, Bonizzoli M, lozzelli D, Migliaccio ML, Zagli G, Bacchereti A, et al. Early intra-intensive care unit psychological intervention promotes recovery from posttraumatic stress disorders, anxiety and depression symptoms in critically ill patients. Crit Care. 2011; 15: 1-8. 
3. Needham DM, Davidson J, Cohen H, Hopkins RO, Weinert C, Wunsch H, et al. Improving longterm outcomes after discharge from intensive care unit: report from a stakeholders' conference. Crit Care Med. 2012; 40: 502-509.

4. Smith TA, Davidson PM, Jenkins CR, Ingham JM. Life behind the mask: The patient experience of NIV. Lancet Respir Med. 2015; 3: 8-10.

5. Arntz A. Imagery rescripting for personality disorders. Cogn Behav Pract. 2011; 18: 466-481.

6. Hilgard ER, Tart CT. Responsiveness to suggestions following waking and imagination instructions and following induction of hypnosis. J Abnorm Psychol. 1966; 71: 196-208.

7. Piccione C, Hilgard ER, Zimbardo PG. On the degree of stability of measured hypnotizability over a 25-year period. J Pers Soc Psychol. 1989; 56: 289-295.

8. Kekecz Z, Varga K. Positive suggestion techniques in somatic medicine: A review of the empirical studies. Interv Med Appl Sci. 2013; 5: 101-111.

9. Karnatovskaia LV, Philbrick KL, Parker AM, Needham DM. Early psychological therapy in critical illness. Semin Respir Crit Care Med. 2016; 37: 136-142.

10. Szilágyi AK., Diószeghy C, Fritúz G, Gál J, Varga K. Shortening the length of stay and mechanical ventilation time by using positive suggestions via MP3 players for ventilated patients. Interv Med Appl Sci. 2014; 6: 3-15.

11. Szilágyi AK, Diószeghy CS, Benczúr L, Varga K. Effectiveness of psychological support based on positive suggestion with the ventilated patient. Eur J Ment Health. 2007; 2: 149-170.

12. Schlanger J, Fritúz G, Varga K. Therapeutic suggestion helps to cut back on drug intake for mechanically ventilated patients in intensive care unit. Interv Med Appl Sci. 2013; 5: 145-152.

13. Maslow AH. A theory of human motivation. Psychol Rev. 1943; 50: 370-396.

14. Teasdale G, Maas A, Lecky F, Manley G, Stocchetti N, Murray G. The Glasgow Coma Scale at 40 years: Standing the test of time. Lancet Neurol. 2014; 13: 844-854.

15. Bradley MM, Lang PJ. Measuring emotion: The self-assessment manikin and the semantic differential. J Behav Ther Exp Psychiat. 1994; 25: 49-59.

16. McKinley S, Coote K, Stein-Parbury J. Development and testing of a Faces Scale for the assessment of anxiety in critically ill patients. J Adv Nurs. 2003; 41: 73-79.

17. Tefikow S, Barth J, Maichrowitz S, Beelmann A, Strauss B, Rosendahl J. Efficacy of hypnosis in adults undergoing surgery or medical procedures: A meta-analysis of randomized controlled trials. Clin Psychol Rev. 2013; 33: 623-636.

18. Faul F, Erdfelder E, Lang A-G, Buchner A. G*power 3: A flexible statistical power analysis program for the social, behavioral, and biomedical sciences. Behav Res Methods. 2007; 39: 175-191. 


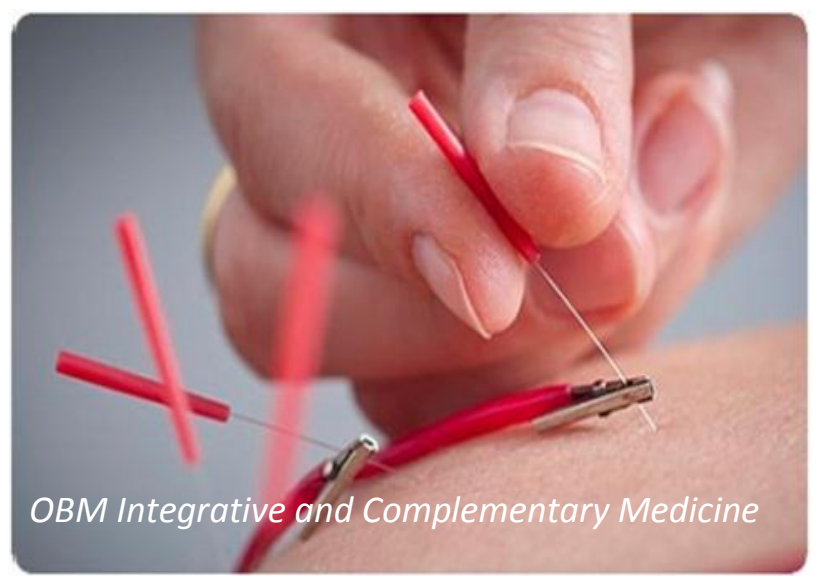

Enjoy OBM Integrative and Complementary Medicine by:

1. Submitting a manuscript

2. Joining in volunteer reviewer bank

3. Joining Editorial Board

4. Guest editing a special issue

For more details, please visit: http://www.lidsen.com/journals/icm 